\title{
A Monte Carlo simulation of scattering reduction in spectral x-ray computed tomography
}

Busi, Matteo; Olsen, Ulrik Lund; Bergbäck Knudsen, Erik; Frisvad, Jeppe Revall; Kehres, Jan; Christensen, Erik D.; Khalil, Mohamad; Haldrup, Kristoffer

Published in:

Advances in Computational Methods for X-Ray Optics IV

Link to article, DOI:

$10.1117 / 12.2273763$

Publication date:

2017

Document Version

Publisher's PDF, also known as Version of record

Link back to DTU Orbit

Citation (APA):

Busi, M., Olsen, U. L., Bergbäck Knudsen, E., Frisvad, J. R., Kehres, J., Christensen, E. D., Khalil, M., \& Haldrup, K. (2017). A Monte Carlo simulation of scattering reduction in spectral x-ray computed tomography. In O. Chubar, \& K. Sawhney (Eds.), Advances in Computational Methods for X-Ray Optics IV (Vol. 10388). [103880P] SPIE - International Society for Optical Engineering. Proceedings of SPIE - The International Society for Optical Engineering https://doi.org/10.1117/12.2273763

\section{General rights}

Copyright and moral rights for the publications made accessible in the public portal are retained by the authors and/or other copyright owners and it is a condition of accessing publications that users recognise and abide by the legal requirements associated with these rights.

- Users may download and print one copy of any publication from the public portal for the purpose of private study or research.

- You may not further distribute the material or use it for any profit-making activity or commercial gain

- You may freely distribute the URL identifying the publication in the public portal 


\section{A Monte Carlo simulation of scattering reduction in spectral $\mathrm{x}$-ray computed tomography}

Matteo Busi, Ulrik L. Olsen, Erik B. Knudsen, Jeppe R. Frisvad, Jan Kehres, et al.

Matteo Busi, Ulrik L. Olsen, Erik B. Knudsen, Jeppe R. Frisvad, Jan Kehres, Erik D. Christensen, Mohamad Khalil, Kristoffer Haldrup, "A Monte Carlo simulation of scattering reduction in spectral x-ray computed tomography," Proc. SPIE 10388, Advances in Computational Methods for X-Ray Optics IV, 103880P (23 August 2017); doi: 10.1117/12.2273763

SPIE Event: SPIE Optical Engineering + Applications, 2017, San Diego, California, United States 


\title{
A Monte Carlo simulation of scattering reduction in spectral X-ray computed tomography
}

\author{
Matteo Busia ${ }^{a}$, Ulrik L. Olsen ${ }^{\mathrm{a}}$, Erik B. Knudsen ${ }^{\mathrm{a}}$, Jeppe R. Frisvad ${ }^{\mathrm{b}}$, Jan Kehres ${ }^{\mathrm{a}}$, Erik D. \\ Christensen $^{\mathrm{c}}$, Mohamad Khalil ${ }^{\mathrm{a}}$, and Kristoffer Haldrup ${ }^{\mathrm{a}}$ \\ ${ }^{a}$ NEXMAP Section, DTU Physics, Fysikvej 307, Kgs. Lyngby, Denmark \\ ${ }^{b}$ DTU Compute, Richard Petersens Plads 324, Kgs. Lyngby, Denmark \\ ${ }^{\mathrm{c}}$ Niels Bohr Institute, Blegdamsvej 17, Copenhagen, Denmark
}

\begin{abstract}
In X-ray computed tomography $(\mathrm{CT})$, scattered radiation plays an important role in the accurate reconstruction of the inspected object, leading to a loss of contrast between the different materials in the reconstruction volume and cupping artifacts in the images. We present a Monte Carlo simulation tool for spectral X-ray CT to predict the scattered radiation generated by complex samples. An experimental setup is presented to isolate the energy distribution of scattered radiation. Spectral CT is a novel technique implementing photon-counting detectors able to discriminate the energy of incoming photons, enabling spectral analysis of X-ray images. This technique is useful to extract efficiently more information on energy dependent quantities (e.g. mass attenuations coefficients) and study matter interactions (e.g. X-ray scattering, photoelectric absorption, etc...). Having a good knowledge of the spectral distribution of the scattered X-rays is fundamental to establish methods attempting to correct for it. The simulations are validated by real measurements using a CdTe spectral resolving detector (Multix ME-100). We observed the effect of the scattered radiation on the image reconstruction, becoming relevant in the energy range where the Compton events are dominant (i.e. above $50 \mathrm{keV}$ ).
\end{abstract}

Keywords: Computed Tomography, Spectral CT, X-ray scattering, Monte Carlo Simulations, Scattered radiation, Fan beam CT

\section{INTRODUCTION}

X-ray computed tomography $(\mathrm{CT})$ is an imaging technique that has been developed and consolidated in last century and is used daily for many purposes primarily medical diagnostics but more and more also other field like airport security screening, food quality, etc. An X-ray CT measurement consists in observing the ratio between the transmitted and incident photons on an object under investigation from multiple angles. It is of key importance to have a good model of the physical interactions between the X-ray photons and the sample. Most of the reconstruction techniques implemented in conventional CT scanners are based on the Lambert-Beer law, ${ }^{1}$ where the interactions are assumed to be fully described by an exponential attenuation model. Moreover, the detectors adopted by these instruments are typically based on single or dual energy methods, meaning that the signal is integrated over broad energy intervals. Consequently, in standard commercial X-ray CT scanners there are two main effects deteriorating the reconstructions.

Firstly, the X-ray source is typically a tube that generates a polychromatic beam, and since the low energy photons are absorbed more efficiently than high energy photons, cupping and streaking artifacts arising in the reconstructions represent an issue known as beam hardening. Monte Carlo simulations have been used to predict and correct such effects ${ }^{2}$ however, it turns out to be challenging in fields where the sample is complex and has a wider range of unknown materials. As an alternative to modeling, one could use spectral photon counting detectors ${ }^{3}$ capable of discriminating the energy of the incoming photons. In this way, under the assumption that the energy resolution is good enough, each measurement can be considered a set of monochromatic acquisitions.

Secondarily, the reconstruction models do not consider that, due to the scattering interactions, the detected total signal $S_{t}(\vec{r}, E)$ is not only composed of the primary radiation $S_{P}(\vec{r}, E)$, which is the photons reaching the

E-mail: mbusi@fysik.dtu.dk; Telephone: +45 71706090 
detector along a linear path, but also by photons having undergone scattering events $S_{s}(\vec{r}, E)$. Johns and Yaffe have shown that the scattering contribution can produce significant cupping artifacts in fan beam geometry CT acquisitions ${ }^{4}$ even though its impact is substantially decreased due to a reduced volume of the sample being irradiated compared to cone beam $\mathrm{CT}$ and the detection solid angle being restricted to the fan beam plane. Only very recently has a way been presented to incorporate the scattered radiation estimate in the forward projection of model based iterative reconstruction techniques to reduce artifacts caused by scattered photons ${ }^{5}$ however, the simulations were carried with a monochromatic beam. A simulation method providing spectral scattering estimate in fast time scales have been developed and validated based on a hybrid Monte Carlo and deterministic approach $^{6}$ thereby introducing a bias in the result. In this work, we present a novel fully stochastic Monte Carlo simulation tool for spectral CT featuring an estimation of the scattered radiation. The tool is validated by comparing simulation results with experimental acquisitions. Finally, the tool is used to predict the scattered contribution of an X-ray spectral CT simulation, and its impact on the slice reconstruction is evaluated.

\section{SIMULATION METHOD}

The simulations were performed using $\mathrm{McXtrace},{ }^{7}$ a software package for Monte Carlo simulation of X-ray experiments by ray-tracing methods. Rather than tracing individual photons, in this framework rays of photons and their interactions are simulated by probabilistic entities defined by weight factors and tracing parameters such as direction, wave-vector, polarization vector, phase etc. The individual parts composing the instrument (e.g. sources, slits, detectors, samples, etc.) are identified as components that can be separately implemented in comparably few lines of simple code. For this work, we have developed a novel sample component suitable for X-ray spectral CT, with explicit treatment of the different physical interactions of X-rays incident on objects composed of multiple materials. The new sample component is initialized by loading a virtual phantom of the object that is made up of a finite number of discretized voxels of a defined size and in a 3D rectangular grid. The value in each voxel holds an integer number $i=0,1,2, \ldots, N$ which labels a specific material. For each material, two different look-up tables are then required. The first look-up table is composed of the energy parametrized photoelectric absorption $\sigma_{p h}^{i}(E)$, coherent (Rayleigh) $\sigma_{c o h}^{i}(E)$ and incoherent (Compton) $\sigma_{i n c}^{i}(E)$ cross sections. These values could for instance be loaded from the database administrated by the National Institute of Standards and Technology (NIST). ${ }^{8}$ This table is used to assess the probability of each interaction.

The second lookup table is used to sample the angular deflection for the scattering events, and is composed of the scattering function $I^{i}(Q)$ of each material, loaded from a database of previously measured X-ray scattering patterns. ${ }^{9}$ In this work, the scattering function is treated as the probability distribution function (PDF) of the scattering vector $\vec{Q}$, of amplitude:

$$
Q=|\vec{Q}|=\frac{4 \pi}{\lambda} \sin \left(\frac{2 \theta}{2}\right)
$$

where $\lambda$ is the wavelength of the incident X-ray and $2 \theta$ is the angle between the incident and the scattered photon. In this way, $Q$ can be sampled by the inversion method, which involves computing the cumulative distribution function $(\mathrm{CDF})$ of the distribution and then inverting that function. Since the model used for the scattering vector is based on experimental data, the distribution is discrete and the computation of the CDF is simply done by adding up the individual probabilities (normalized to sum 1) for the various points of the distribution. The scattering angle $2 \theta$ is then obtained from the sampled $Q$ using eq. 1 since the energy of the incident ray is a known parameter. A limitation of the current implementation is that the angular sampling is treated the same way for both Rayleigh and Compton scattering events. As an alternative, one could use analytical approximations for the incoherent scattering as presented by Hajdu ${ }^{10}$ and Palinkas, ${ }^{11}$ or sample the angular deviation as predicted by the Thomson and Klein-Nishina functions. ${ }^{12}$ The azimuthal angle $\phi$ is uniformly sampled in the interval $(0,2 \pi)$, since the source emitted by a conventional X-ray tube is considered to be randomly polarized. Finally, if the scattering event is determined to be of Compton type, the ray's energy parameter is updated to $E_{\text {inc }}$ according to the Compton energy shift relation: ${ }^{13}$

$$
E_{\text {inc }}=\frac{E}{1+\frac{E}{511 \mathrm{keV}}(1-\cos (\theta))}
$$

Figure 1 shows an example of the look-up tables for $\mathrm{H}_{2} \mathrm{O}$ read by the component to simulate the interactions. The pseudo-code of the component is shown in Algorithm 1. Therein, the step length $s_{l}$ represents the rate at 
which the interaction probabilities are checked while the ray is traced through the sample up to the final length $l$. In the following simulations $l$ is set to be to be four times smaller than the voxel size, except at the first step of each ray traced, where the step length is multiplied by a random generated number to reduce artifacts induced by the regularity. ${ }^{14} \mu(E)$ represent the photoelectric absorption linear attenuation, while $\rho^{i}$ and $\sigma_{p h}^{i}(E)$ are respectively the density relative to water and the photoelectric absorption cross section of each material.

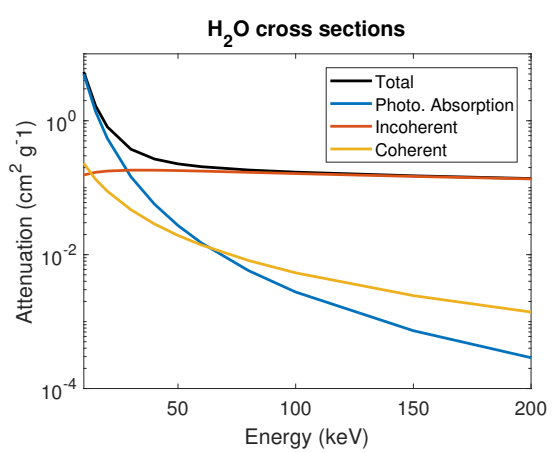

(a)

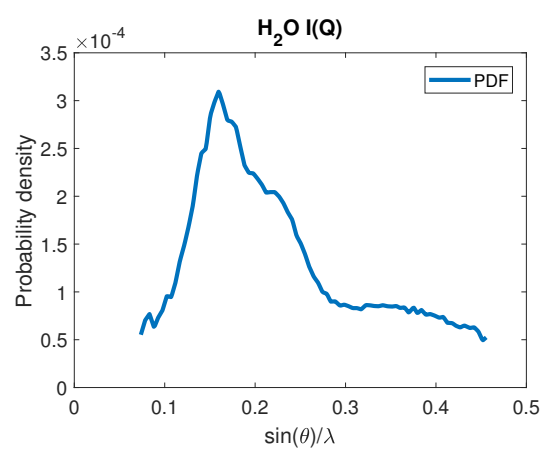

(b)

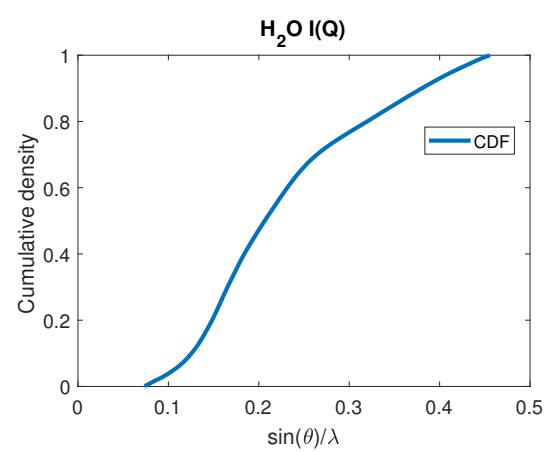

(c)

Figure 1: The water look-up tables used by the sample component. In (a) the NIST cross sections are represented for each type of interaction: photoelectric absorption (blue), Rayleigh scattering (yellow), Compton scattering (red) and total attenuation (black), that is the sum of the previous three. Note the logarithmic scale to emphasize each contribution to the total attenuation. Above $50 \mathrm{keV}$, Compton scattering is dominant whereas photoelectric absorption is prevailing for low energies. Rayleigh scattering is about two orders of magnitude lower than the total attenuation. In (b) and (c), the water scattering function's probability distribution function (PDF) and its respective cumulative distribution function $(\mathrm{CDF})$ respectively are represented.

Data: Object's phantom and material densities $\rho^{i}$, cross sections $\sigma^{i}(E)$ and scattering functions $I^{i}(Q)$

Result: Energy resolved total and scattering signal following the interaction between X-rays and the object

initialize system's geometry and variables;

set the step length $s_{l}$;

while ray is within the object do

if ray is scattered then

sample scattering angle $\theta$;

if Compton scattering then

update to Compton energy with eq. 2;

update ray direction according to $\theta$;

else

Rayleigh scattering;

update ray direction according to $\theta$;

end

end

read photoelectric absorption linear attenuation $\mu(E)=\rho^{i} \sigma_{p h}^{i}(E)$;

ray travels the step length $s_{l}$;

increment the cumulative linear attenuation along the trace $\mu(E) l=\mu(E) l+\mu(E) s_{l}$;

end

apply final weight to the ray: $e^{-\mu(E) l}$;

Algorithm 1: Pseudo-code of the McXtrace object component. 


\section{EXPERIMENTAL VALIDATION}

In order to validate the simulation tool we have designed an instrumental setup to detect the isolated radiation being scattered from the sample. The X-ray beam was generated by a tungsten anode and the acceleration voltage and filament current were set to $150 \mathrm{keV}$ and $0.5 \mathrm{~mA}$ respectively. For these operating parameters, the focal spot was $75 \mu \mathrm{m}$. A $3 \mathrm{~mm}$ thick aluminum filter was placed in front of the source to suppress the low energy characteristic fluorescent lines emitted by the source, in order to reduce the beam hardening effects and the detection of photons with energy below the lowest threshold of the detector. The detector was a spectral photon counting 1D detector, namely Multix-ME100, ${ }^{3}$ composed of $1 \times 256$ square pixels of pitch $0.8 \mathrm{~mm}$ with 128 energy bins of width $1.1 \mathrm{keV}$, linearly distributed between 20 and $160 \mathrm{keV}$. An energy distribution of the source was obtained by an acquisition of the flat field (i.e. without the sample being inserted) and was used as the probability distribution of the ray's energy parameter for the simulated source. Figure 2(a) shows the spectral shape generated by the X-ray source in both experiment and simulations. The detector spectral effects (e.g. escape peaks, charge sharing, pile-up, etc.) of the acquired source spectrum were corrected for by using a method developed by Christensen et al. ${ }^{15}$ so that the detector could be considered ideal in the simulations. As an alternative, one would have to apply a detector response matrix to the results. ${ }^{16}$ The X-ray beam was collimated into a fan beam geometry, whereas the linear detector was sequentially translated vertically to reproduce a $70 \times 2562$ D-detector. To isolate the radiation contribution being scattered from the sample $S_{s}(\vec{r}, E)$ to the total signal $S_{t}(\vec{r}, E)=S_{p}(\vec{r}, E)+S_{s}(\vec{r}, E)$, we analyzed the signal detected in the region outside of the fan beam plane. The sample used in the experiments was made up of four glass $\left(\mathrm{SiO}_{2}\right)$ bottles filled with water $\left(\mathrm{H}_{2} \mathrm{O}\right)$, hydrogen peroxide $\left(\mathrm{H}_{2} \mathrm{O}_{2}\right)$, powdered sugar $\left(\mathrm{C}_{12} \mathrm{H}_{22} \mathrm{O}_{11}\right)$, a powdered PETN explosive simulant $\left(\mathrm{C}_{5} \mathrm{H}_{8} \mathrm{~N}_{4} \mathrm{O}_{12}\right)$ and an aluminum rod. The sample was designed to expose the challenge of accurate classification of materials with very similar properties. A sketch of the experimental setup is shown in figure 2(b).

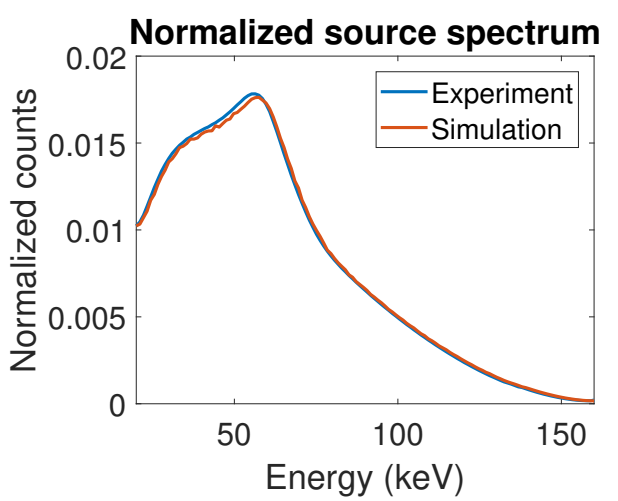

(a)

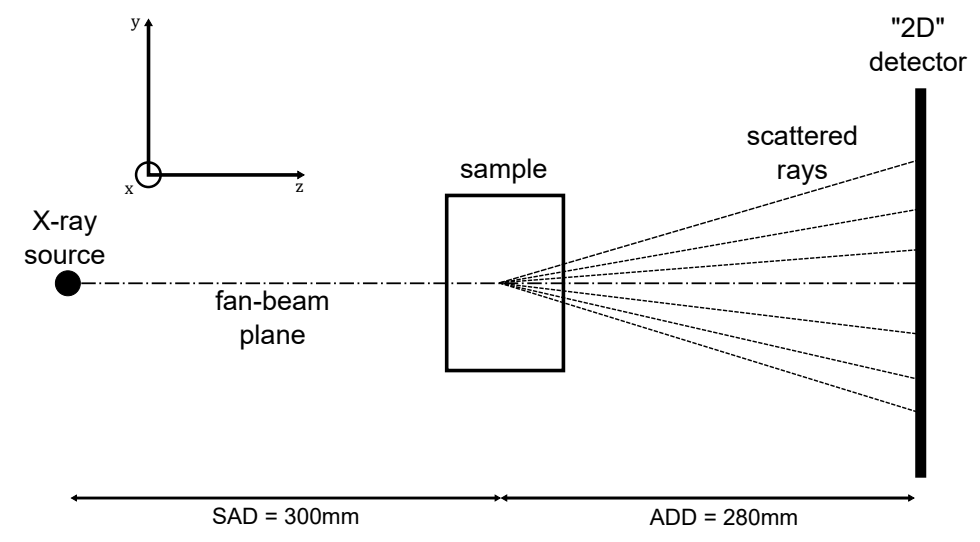

(b)

Figure 2: (a) Source spectrum shape obtained by the acquisition of the flat field (blue line) and by the simulation (red line). (b) Experimental sketch of the setup. The X-ray beam was collimated into a fan beam geometry. The sample, placed at a distance $\mathrm{SAD}=300 \mathrm{~mm}$ from the detector, was composed of four glass bottles filled with different materials and an aluminum rod. The $1 \mathrm{D}$ detector, placed at a distance $\mathrm{ADD}=280 \mathrm{~mm}$ from the sample, was composed of 256 pixels in the $x$ direction and is vertically translated in the $y$ direction to reproduce a $2 \mathrm{D}$ detector. At the detector surface not hit by the fan beam plane, the signal consists of radiation being scattered by the sample.

A simulation of the identical setup was performed to compare the results. A mathematical phantom of the sample of $150 \times 150$ voxels of size $0.667 \mathrm{~mm}$ was generated and used as an input for the Monte Carlo simulations. The simulation runtime for a single projection with $10^{7}$ rays being sampled was 13 minutes on a standard laptop. The performance is similar and in some instances faster than in some previous recent works ${ }^{6}$ for a 
sample of similar geometrical size. For CT simulations, several projections can be simulated in parallel in multicore architectures to increase efficiency. The output of the simulation, showing the distinct contribution of the Rayleigh and Compton scattering to the total signal is shown in figure 3, where the energy channels has been merged in two intervals. The first in the low energy range $[20,54.2] \mathrm{keV}$ and the second in the high energy range $[125.8,160] \mathrm{keV}$. As expected, the scattering angle increases as the energy decreases, indicating that information about the geometrical structure of the sample is lost at low energies. A similar behavior, shown in figure 4, is found by comparing the contribution from single and multiple scattering (i.e. when then ray scatters respectively once and more than once in the same tracing). For multiple scattered rays, the features of the sample are lost and the rays are randomly distributed around the center of the sample. Furthermore, the Compton scattered signal becomes particularly relevant at high energies where a loss of contrast between the different parts of the samples is visible by comparing figures $3(\mathrm{a})$ and $3(\mathrm{~d})$. It should be noted that the simulation output is on an arbitrary scale since it is a probability map. Therefore, the data should be interpreted after having rescaled it properly according to the ray flux however, in most of applications this procedure is not necessary since the reconstruction techniques typically require the data set to be normalized as attenuation $A(\vec{r}, E)$ according to eq:

$$
A(\vec{r}, E)=-\log \frac{S(\vec{r}, E)}{S_{0}(\vec{r}, E)}
$$

where $S(\vec{r}, E)$ and $S_{0}(\vec{r}, E)$ are the detected signal at the pixel position $\vec{r}$ and at the energy channel $E$, respectively with and without the sample being inserted.

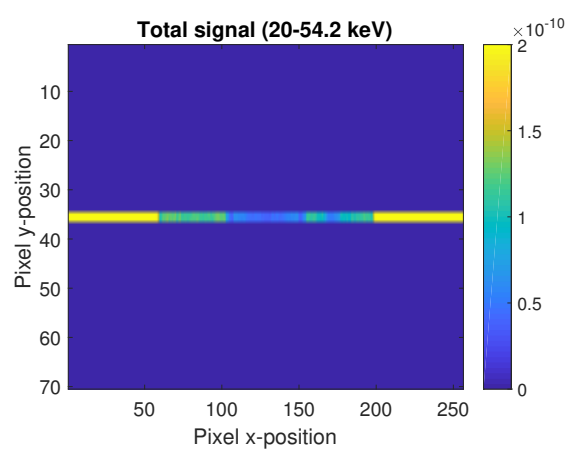

(a)

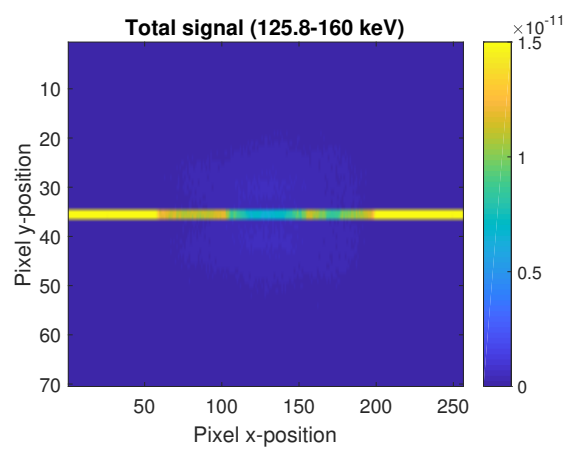

(d)

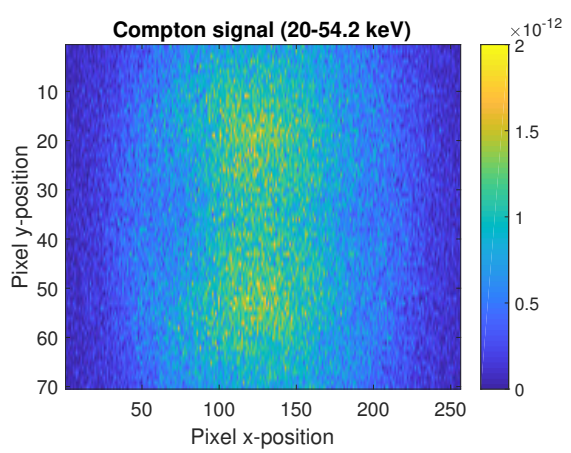

(b)

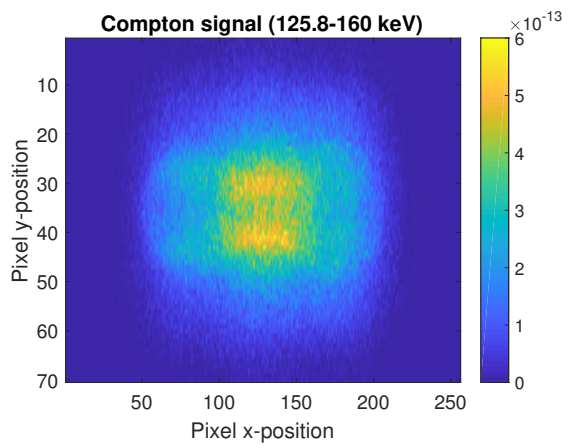

(e)

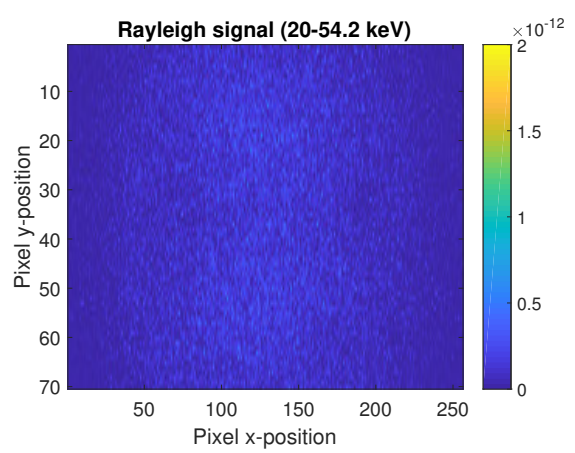

(c)

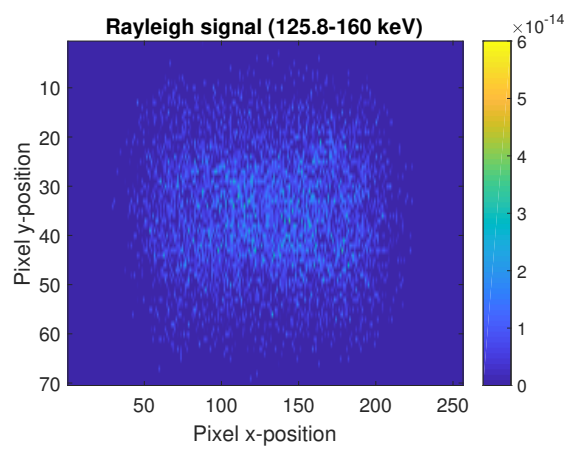

(f)

Figure 3: Simulation output of the total signal $S_{t}(\vec{r}, E)$ (a,d) and the Compton (b,e) and Rayleigh (c,f) contribution to the scattered signal $S_{s}(\vec{r}, E)$, obtained by merging the energy channels between 20.0 and $54.2 \mathrm{keV}$ $(\mathrm{a}, \mathrm{b}, \mathrm{c})$ and between 125.8 and $160 \mathrm{keV}(\mathrm{d}, \mathrm{e}, \mathrm{f})$. The fan beam plane is clearly visible in (a,d), represented by the central horizontal line. Note the color scale of $(b, c)$ is two order of magnitude lower than (a), similarly the color scale of (e) and (f) is respectively 1.4 and 2.4 orders of magnitude lower than (d). 


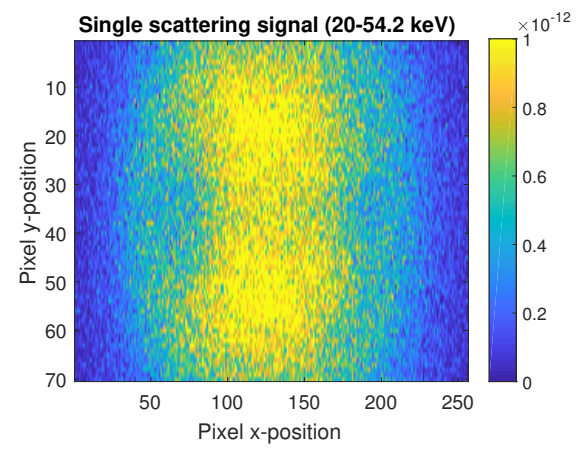

(a)

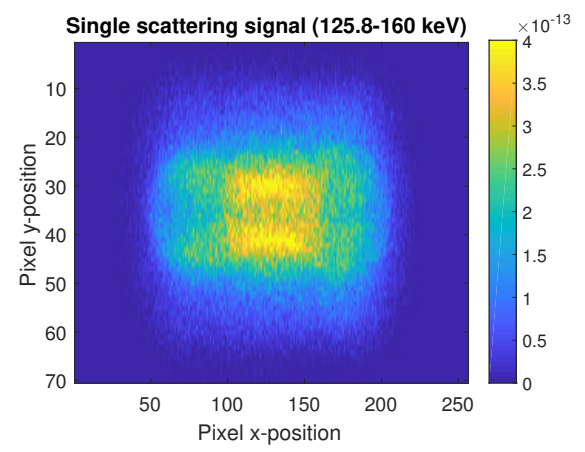

(c)

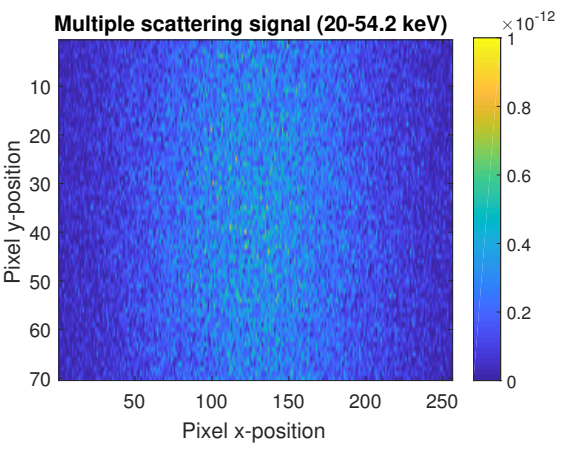

(b)

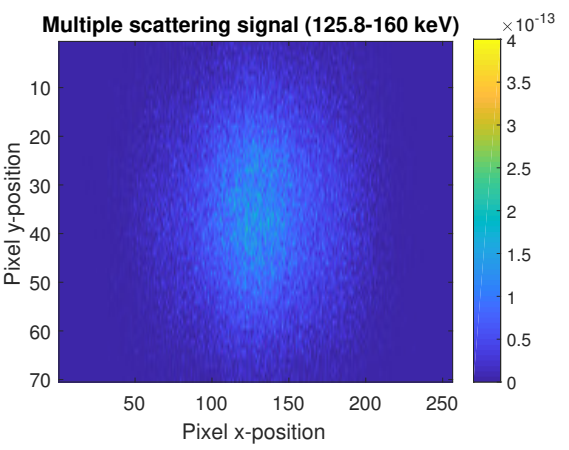

(d)

Figure 4: Simulation output of the single $(\mathrm{a}, \mathrm{c})$ and the multiple $(\mathrm{b}, \mathrm{d})$ scattering signal $S_{s}(\vec{r}, E)$ obtained by merging the energy channels between 20.0 and $54.2 \mathrm{keV}(\mathrm{a}, \mathrm{b})$ and between 125.8 and $160 \mathrm{keV}(\mathrm{c}, \mathrm{d})$.

Figure 5 displays the comparisons of the scattering data obtained by both the simulation and experiment. In figure 5(a), the horizontal profile of the scattered radiation is represented. Rather than analyzing singularly each horizontal line, we have integrated the signal in the area out of the fan beam plane to have better statistics. For the same purpose, all the energy channels have been integrated since we have previously discussed the behavior of the spatial distribution as function of the energy. The energy distribution shape of the scattered signal is shown in figure5(b). It is evident by comparing the two lines in the figures that the simulation underestimates the high energy, and thus supposedly the incoherent (Compton), scattering events. This can be caused, for instance, by the impact of the background radiation incoming from the environment, due to the difficulties of achieving a perfect fan-beam collimation. The consequence is a mismatch in the spatial profile, since the model that has been used for the angular sampling of the scattering events does not distinguish by the event type. We expect that by using a separate model for the incoherent scattering, as discussed in the previous section, the sampled deflection will be shifted towards higher angles converging to the results obtained by the experiments. Another reason inducing the discrepancy is that the $Q$-range used for the sampling is defined experimentally therefore is limited by the instrumental constraints as seen in figure 1(b). Consequently, the very high and low scattering angles are not included in the simulations.

\section{TOMOGRAPHY SIMULATION}

In the last part of the work, we have simulated a tomography scan of 37 projections linearly distributed between $\omega=(0,2 \pi)$. The specifications of the instrument components and of the sample are as described in the previous section. The reconstruction technique adopted was the one presented Sidky and Pan, ${ }^{17}$ namely an algebraic reconstruction technique (ART) with total variation (TV) regularization, that has been proven to be particularly efficient for limited views datasets. To assess the impact of the scattered radiation, we have performed slice reconstructions of the total attenuation $A_{t}(\vec{r}, E)$ and of the primary attenuation $A_{p}(\vec{r}, E)$. These are obtained 


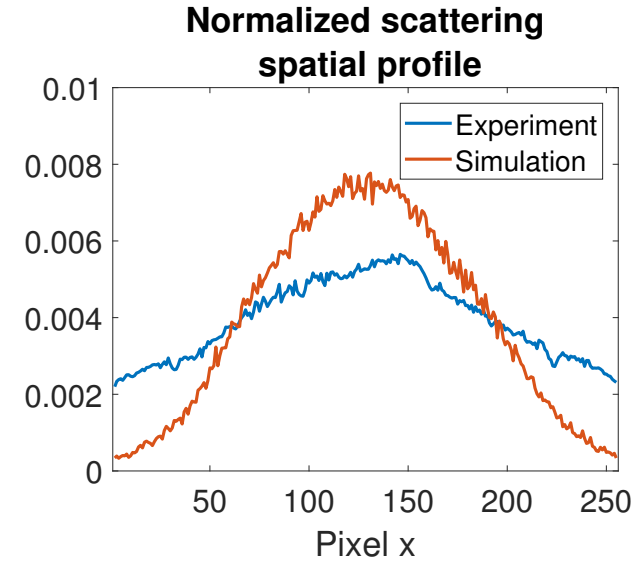

(a)

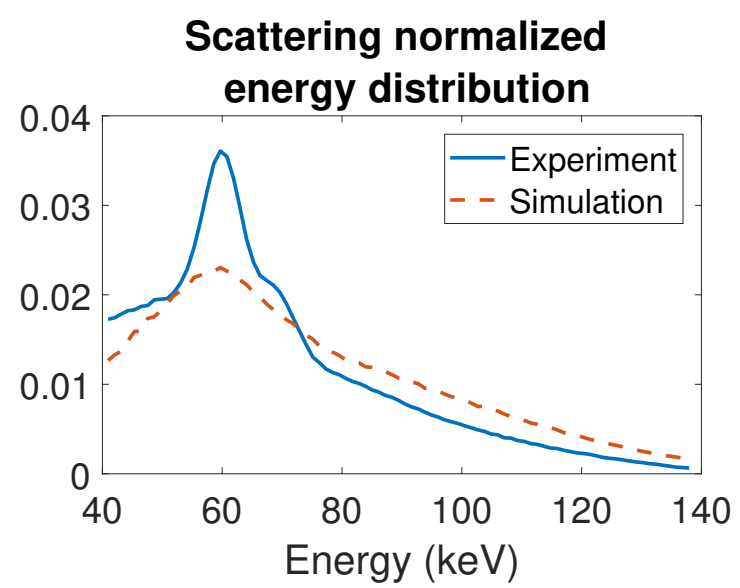

(b)

Figure 5: In (a) the normalized spatial profile of the scattered radiation is obtained by merging the y-pixels out of the fan beam plane. Its respective normalized energy distribution is shown in (b).

by inserting respectively the total signal $S_{t}(\vec{r}, E)$ and the primary radiation $S_{p}(\vec{r}, E)=S_{t}(\vec{r}, E)-S_{s}(\vec{r}, E)$ in eq. 3. The datasets have been divided in four energy ranges combining the energy channels in the intervals $E=\{[20,54.2],[55.3,89.5],[90.6,124.7],[125.8,160]\} \mathrm{keV}$ however, we only discuss the results for the latter. In figure 6 , the slice reconstruction result of the primary attenuation is shown. The result is compared to the total attenuation reconstruction using the uncorrected total signal, by taking the residual between the two (i.e. $\left.A_{p}(\vec{r}, E)-A_{t}(\vec{r}, E)\right)$. It is found that the correction, in high energy regimes, accounts for a relative increase in amplitude of the reconstructed attenuation up to $8 \%$. More importantly, the correction gain is different for each material being largest for the powdered sugar, which is expected to be the sample type giving rise to most scattering due to its crystallinity. Therefore, we expect the correction to be relevant to improve the contrast between different materials, for acquisitions at high energy regimes. Finally, it can be seen in figure 6(b) that the correction diminish the cupping artifacts in the reconstruction, as the residual gets larger towards the center of the bottle.

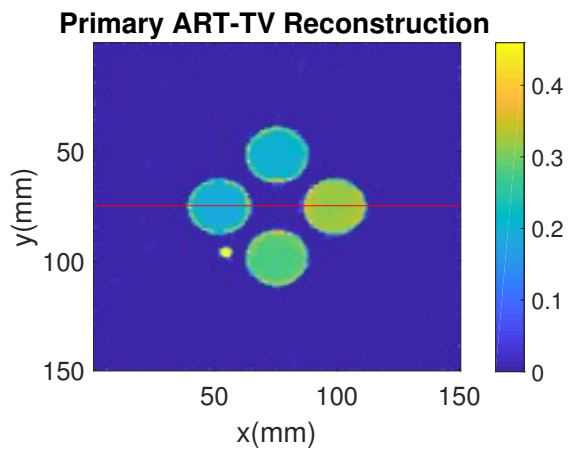

(a)

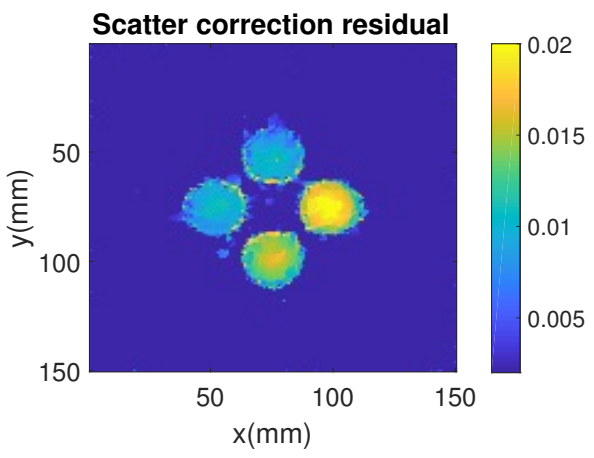

(b)

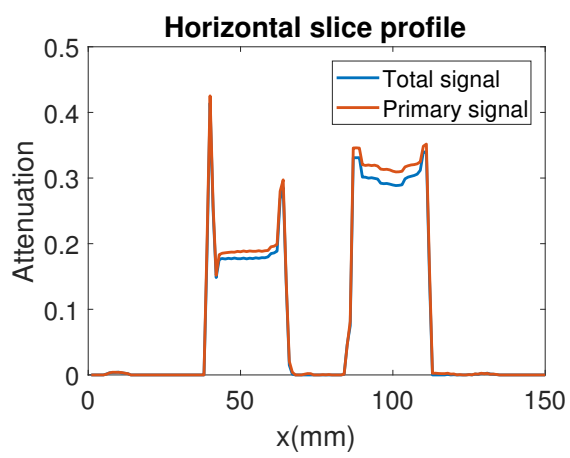

(c)

Figure 6: (a) The slice reconstruction of the primary attenuation $A_{p}(\vec{r}, E)$. The overlapping red line shows the horizontal line corresponding to the profile plotted in (c). In the latter the blue line is the result of the total attenuation $A_{t}(\vec{r}, E)$, while the red line is the result of the primary attenuation $A_{p}(\vec{r}, E)$ obtained by the subtraction of the scattering contribution from the total signal. The residual of the two reconstructions (namely of $A_{p}(\vec{r}, E)$ and $\left.A_{t}(\vec{r}, E)\right)$ is displayed in (b). 


\section{CONCLUSIONS}

We have presented a fully stochastic simulation tool for spectral X-ray CT, featuring an estimate of the scattered radiation of fast computational speed. It has been validated by an experiment designed to detect the isolated radiation being scattered by the sample. We have discussed the current limitations of the simulation tool, and possible strategies to overcome them. In a preliminary approach, we have shown how the scattering estimate can improve the attenuation reconstruction by a restoration of the primary radiation $S_{p}(\vec{r}, E)$, performed as a simple subtraction of the scattering contribution $S_{s}(\vec{r}, E)$. It is found that especially at high energies, where the Compton scattering events are dominant, the correction is useful to improve the contrast between different materials. This is expected to aid the automated segmentation procedures leading to a more accurate material classification. In future work, we aim to incorporate the scattering estimation in the forward model of a model based iterative reconstruction (MBIR) technique, to improve even further the efficiency of the algorithm.

\section{ACKNOWLEDGMENTS}

The Authors would like to acknowledge Innovation Fund Denmark for financing their respective work.

\section{REFERENCES}

[1] Swinehart, D., "The Beer-Lambert law," J. Chem. Educ 39(7), 333 (1962).

[2] Thomsen, M., Knudsen, E. B., Willendrup, P. K., Bech, M., Willner, M., Pfeiffer, F., Poulsen, M., Lefmann, K., and Feidenhans, R., "Prediction of beam hardening artefacts in computed tomography using Monte Carlo simulations," Nuclear Instruments and Methods in Physics Research Section B: Beam Interactions with Materials and Atoms 342, 314-320 (2015).

[3] Gorecki, A., Brambilla, A., Moulin, V., Gaborieau, E., Radisson, P., and Verger, L., "Comparing performances of a CdTe X-ray spectroscopic detector and an X-ray dual-energy sandwich detector," Journal of Instrumentation 8(11), P11011 (2013).

[4] Johns, P. C. and Yaffe, M., "Scattered radiation in fan beam imaging systems," Medical physics 9(2), 231-239 (1982).

[5] Dremel, K. and Fuchs, T., "Scatter simulation and correction in computed tomography: A reconstructionintegrated approach modelling the forward projection," NDT $\mathscr{E}$ E International 86, 132-139 (2017).

[6] Sossin, A., Tabary, J., Rebuffel, V., Ltang, J., Freud, N., and Verger, L., "Fast scattering simulation tool for multi-energy X-ray imaging," Nuclear Instruments and Methods in Physics Research Section A: Accelerators, Spectrometers, Detectors and Associated Equipment 802, 60 - 66 (2015).

[7] Bergbäck Knudsen, E., Prodi, A., Baltser, J., Thomsen, M., Kjær Willendrup, P., Sanchez del Rio, M., Ferrero, C., Farhi, E., Haldrup, K., Vickery, A., et al., "McXtrace: a Monte Carlo software package for simulating X-ray optics, beamlines and experiments," Journal of Applied Crystallography 46(3), 679-696 (2013).

[8] Berger, M. J., Hubbell, J., Seltzer, S., Chang, J., Coursey, J., Sukumar, R., Zucker, D., and Olsen, K., "XCOM: Photon cross sections database," NIST Standard reference database 8(1), 3587-3597 (1998).

[9] Kehres, J., Lyksborg, M., and Lund Olsen, U., "Threat detection of liquid explosives and precursors from their X-ray scattering pattern using energy dispersive detector technology," Submitted to Optics and Photonics 2017: X-ray, Gamma-ray, and Particle Technologies (2017).

[10] Hajdu, F., "Revised parameters of the analytic fits for coherent and incoherent scattered X-ray intensities of the first 36 atoms," Acta Crystallographica Section A: Crystal Physics, Diffraction, Theoretical and General Crystallography 28(3), 250-252 (1972).

[11] Palinkas, G., "Analytic approximations for the incoherent X-ray intensities of the atoms from Ca to Am," Acta Crystallographica Section A: Crystal Physics, Diffraction, Theoretical and General Crystallography 29(1), 10-12 (1973).

[12] Baró, J., Roteta, M., Fernández-Varea, J., and Salvat, F., "Analytical cross sections for Monte Carlo simulation of photon transport," Radiation physics and chemistry 44(5), 531-552 (1994).

[13] Compton, A. H., "A quantum theory of the scattering of X-rays by light elements," Physical review 21(5), $483(1923)$. 
[14] Pauly, M., Kollig, T., and Keller, A., "Metropolis light transport for participating media," in [Rendering Techniques 2000 (Proceedings of EGWR 2000)], 11-391, Springer (2000).

[15] Dreier Christensen, E., Gu, Y., Kehres, J., Feidenhansl, R., and Lund Olsen, U., "Spectral correction algorithm for multispectral CdTe X-ray detectors," Submitted to Optics and Photonics 2017: X-ray, Gamma-ray, and Particle Technologies (2017).

[16] Bootsma, G., Verhaegen, F., and Jaffray, D., "Spatial frequency spectrum of the X-ray scatter distribution in CBCT projections," Medical physics 40(11) (2013).

[17] Sidky, E. Y., Kao, C.-M., and Pan, X., "Accurate image reconstruction from few-views and limited-angle data in divergent-beam CT," Journal of X-ray Science and Technology 14(2), 119-139 (2006). 